\title{
Mathematical Modeling and Characteristic Analysis of the Vertical Stiffness for Railway Vehicle Air Spring System
}

\author{
Liufeng Xu (iD \\ School of Traffic and Transportation Engineering, Central South University, Changsha, Hunan 410075, China \\ Correspondence should be addressed to Liufeng Xu; xuliufengxd@csu.edu.cn
}

Received 26 September 2019; Revised 21 March 2020; Accepted 6 May 2020; Published 8 June 2020

Academic Editor: Gianluca Gatti

Copyright $\odot 2020$ Liufeng Xu. This is an open access article distributed under the Creative Commons Attribution License, which permits unrestricted use, distribution, and reproduction in any medium, provided the original work is properly cited.

Establishing a correct and reliable vertical stiffness model has an important significance on reproducing the characteristics of an air spring system. In this paper, a dynamic vertical stiffness model is developed based on thermodynamics and fluid dynamics, and geometric parameters are identified by an approximate analytical method. Meanwhile, experimental tests are performed to verify the accuracy and reliability of the proposed model. Furthermore, the impact of geometric parameters on the vertical stiffness characteristics is discussed through a sensitivity analysis. The conclusions show that the dynamic vertical stiffness model can well characterize the dynamic characteristics of the air spring system, which provides a theoretical basis for the optimal design of air spring parameters and the study of mechanical properties.

\section{Introduction}

With significant advantages of vibration isolation performance, air spring suspension systems have been extensively applied in urban rail vehicles, high-speed trains, and commercial vehicles [1]. The air spring effectively attenuates harmful vibration and improves the running stability and ride comfort of vehicles. Establishing a correct and reliable model has an important significance on reproducing the characteristics of the air spring system and improving the stability and ride comfort of vehicles.

In metro vehicles, the air spring is a free membrane type air spring located between the car body and bogie frame, as shown in Figure 1.

The free membrane type air spring is a typical belloworifice-chamber pneumatic system; it consists of a rubber diaphragm, an auxiliary chamber, and an orifice. Due to the influence of elastic materials, geometry, and constraints, the vertical characteristics of the air spring show strong nonlinearity. Therefore, establishing a precise vertical model is the key to investigate the characteristics of the air spring system and analyze the influence of geometric parameters on the stiffness performance and vehicle ride comfort.
Enormous efforts have been devoted by many scholars to the vertical stiffness modeling and geometric parameter optimization analysis of the air spring system. Alonso et al. [2] established a test bench to describe the characteristics of the air spring system and explored the relationship between the geometric parameters and ride comfort. Genovese et al. [3] developed a numerical finite element model to analyze the behavior of air spring. Shi et al. [4] built a finite element model of an air spring to study the static elastic characteristics and utilized sensitivity analysis method to study the impact of different parameters on the elastic characteristic. Li et al. [5, 6] and Chen et al. [7] deduced an analytical formula to study air spring vertical stiffness, and the influence of geometric parameters on vertical stiffness was analyzed. Liu and Lee [8] derived a theoretical model based on the energy conservation equation and gas state equation, to investigate the dynamic stiffness and overall equivalent damping of air spring. Quaglia et al. [9, 10] proposed a dimensionless linear model for pneumatic suspension based on hydrodynamics and offered some design considerations to fully understand the effect of parameters on the stiffness characteristics. Docquier et al. [11] presented several models of the bellow-pipe-tank pneumatic suspension subsystem, and each element was analyzed to produce a global model for 


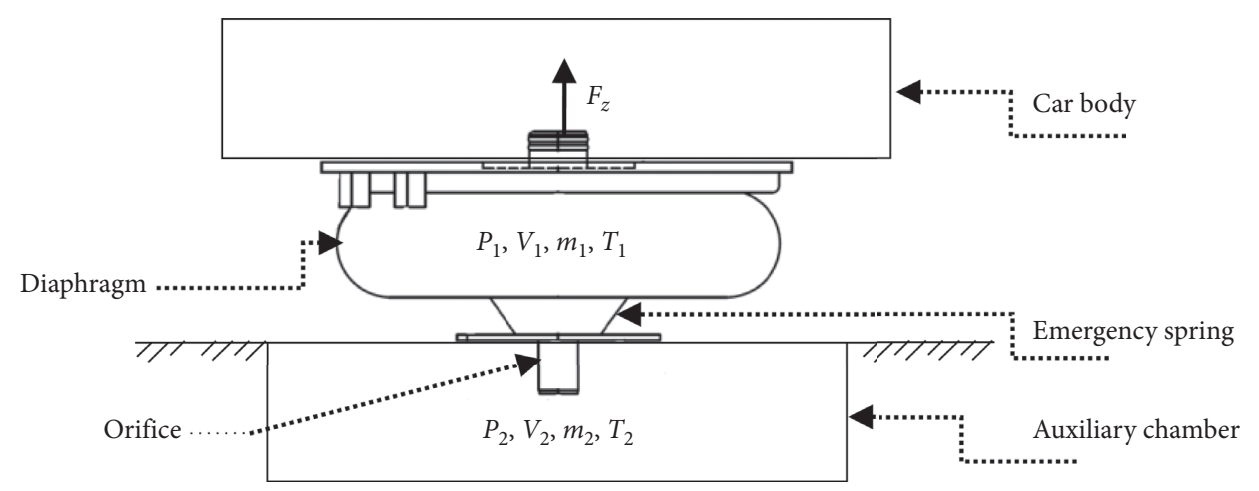

FIGURE 1: Simplified structure of air spring.

a railway vehicle. Nakajima et al. [12] developed a nonlinear model of air spring system coupling with leveling valves and differential pressure valves based on thermodynamics to reflect air spring nonlinear behaviors. Zhu et al. [13] developed a vertical dynamic model to predict the dynamic characteristics of a bellow-pipe-tank pneumatic subsystem air spring based on thermodynamics, effective friction, and viscoelastic damping of the bellow rubber. Facchinetti et al. [14] developed two different modeling approaches for air sprig suspension, a quasistatic one based on mechanics analysis and a dynamic one based on thermodynamics. Wang and Zhu [15] derived a nonlinear stiffness model of air spring based on hydrodynamics and thermodynamics, and the nonlinear model was processed with a linearising method of small deviation based on the Taylor series.

As can be seen from the above references, various methods have been adopted to study the air spring vertical stiffness model and its mechanical properties, especially on the bellowpipe-tank air spring system, but few studies are concerned with the vertical stiffness modeling for rubber diaphragm-orificeauxiliary chamber air spring system. In addition, in previous studies, the structural parameters of air spring were determined from experiments, which was unacceptable for dynamic stiffness modeling and structural optimization design.

Therefore, the purpose of this paper is to establish a dynamic vertical stiffness model for rubber diaphragm-orifice-auxiliary chamber air spring system on the basis of thermodynamics and fluid dynamics, and the analytical solutions of the effective area, the equivalent volume, and their derivative terms are obtained by an approximate analytical method.

The outline of this study is as follows: firstly, a dynamic vertical stiffness model and a restriction coefficient model were constructed based on thermodynamics and fluid dynamics, and the analytical solutions of the effective area, the equivalent volume, and its derivative terms are obtained by the approximate analytical method. Secondly, a series of air spring dynamic tests were carried out to verify the accuracy and reliability of the proposed dynamic vertical stiffness model. Meanwhile, the impact of the geometric parameters on the vertical stiffness characteristics of the air spring was discussed with a sensitivity analysis method. Finally, a research conclusion showed that the dynamic vertical stiffness model can accurately predict the mechanical characteristics of the air spring system, and some valuable suggestions were obtained, which can provide guidance for air spring parameter optimization design and mechanical performance improvement.

\section{Mode Development}

Establishing a precise model of the metro vehicle air spring, the stiffness model of rubber diaphragm and auxiliary chamber, and the damping model of orifice connecting the diaphragm with auxiliary chamber must be considered. In view of previous researches $[16,17]$, the air spring vertical stiffness model and the restriction coefficient model of the orifice are established based on thermodynamics and fluid dynamics. Furthermore, the variable geometric parameters such as the effective area, the equivalent volume, and their derivative terms are solved by an approximate analytical method.

2.1. Vertical Stiffness Modeling. The pneumatic system of air spring is a closed environment, the internal temperature and heat meet the thermodynamics law, and the gas in the pneumatic system can be considered as an ideal gas. Referring to literature $[9,13,18]$, the relations of pressure, volume, and temperature in the air spring rubber diaphragm can be expressed by the ideal gas state equation obtained by Boyle-Charles law:

$$
P_{1} V_{1}=\frac{m_{1}}{M_{1}} G T_{1}
$$

where $P_{1}, V_{1}, m_{1}$, and $T_{1}$ are the inner pressure, volume, air mass, and temperature in the air spring rubber diaphragm, respectively. $M_{1}$ is the molar mass of gas, $G$ is the molar gas constant, $G=287.1 \mathrm{~J} /(\mathrm{kg} \cdot \mathrm{K})^{-1}$, and the initial air temperature $T_{1}=293 \mathrm{~K}$.

The change process of gas in the air spring can be regarded as an isentropic process and described by the polytropic equation:

$$
\begin{gathered}
P_{1}\left(\frac{V_{1}}{m_{1}}\right)^{n}=P_{10}\left(\frac{V_{10}}{m_{10}}\right)^{n}, \\
\frac{T_{1}}{T_{10}}=\left(\frac{P_{1}}{P_{10}}\right)^{((n-1) / n)},
\end{gathered}
$$


where the subscript " 0 " indicates the initial value and $n$ is the polytropic coefficient within the range of 1-1.4, which represents an isothermal process and an adiabatic process, respectively.

Differentiating (2) and combining (1) and (3) yield the time variation of the internal pressure of rubber diaphragm:

$$
\dot{P}_{1}=-\frac{n G T_{10}}{V_{1}}\left(\frac{P_{1}}{P_{10}}\right)^{((n-1) / n)} \dot{m}_{1}-\frac{n P_{1}}{V_{1}} \dot{V}_{1} .
$$

Based on the continuity equation [19], the time derivative of the air mass in the rubber diaphragm is the mass flow rate, and it is given as

$$
\dot{m}_{1}=-\frac{\mathrm{d} m_{1}}{\mathrm{~d} t}=-\frac{\mathrm{d}\left(\rho_{1} V_{1}\right)}{\mathrm{d} t}=-\frac{\mathrm{d} \rho_{1}}{\mathrm{~d} t} V_{1}-\frac{\mathrm{d} V_{1}}{\mathrm{~d} t} \rho_{1},
$$

where $\rho_{1}$ is the air density in rubber diaphragm.

The volume $V_{1}$ is mainly related to the change of internal pressure $\dot{P}_{1}$ and vertical displacement $x$; the change rate of the rubber diaphragm volume $\dot{V}_{1}$ can be expressed as

$$
\dot{V}_{1}=\frac{\partial V_{1}}{\partial x} \dot{x}+\frac{\partial V_{1}}{\partial P_{1}} \dot{P}_{1}
$$

Similarly, the change rate of air spring effective area $\dot{A}_{\text {ef }}$ is expressed as

$$
\dot{A}_{\mathrm{ef}}=\frac{\partial A_{\mathrm{ef}}}{\partial x} \dot{x}+\frac{\partial A_{\mathrm{ef}}}{\partial P_{1}} \dot{P}_{1} .
$$

The air spring elastic force $F_{Z}$ in the vertical direction provided by the compressed air in the rubber diaphragm is expressed as

$$
F_{Z}=\left(P_{1}-P_{a}\right) A_{\mathrm{ef}},
$$

where $P_{a}$ is the atmospheric pressure.

A model of the auxiliary chamber can be obtained simply by writing the continuity equation for chamber volume $V_{2}$ in the same way as for the rubber diaphragm. The difference is that the auxiliary chamber volume $V_{2}$ is constant. The change of pressure in the auxiliary chamber with time can be similarly expressed as

$$
\dot{P}_{2}=-\frac{n G T_{20}}{V_{2}}\left(\frac{P_{2}}{P_{20}}\right)^{((n-1) / n)} \dot{m}_{2}
$$

where $P_{2}, V_{2}, m_{2}$, and $T_{2}$ are the internal pressure, volume, air mass, and temperature in the auxiliary chamber, respectively.

The air flow entering the auxiliary chamber is expressed as

$$
\dot{m}_{2}=\frac{\mathrm{d} m_{2}}{\mathrm{~d} t}=\frac{\mathrm{d}\left(\rho_{2} V_{2}\right)}{\mathrm{d} t}=\frac{\mathrm{d} \rho_{2}}{\mathrm{~d} t} V_{2} .
$$

The vertical stiffness of air spring $K_{\mathrm{dyn}}$ can be defined as the derivative of the elastic force $F_{Z}$ with respect to the vertical displacement $x$ :

$$
\begin{aligned}
K_{\mathrm{dyn}}= & -\frac{\mathrm{d} F_{z}}{\mathrm{~d} x}=\frac{n G T_{10}}{V_{1}} A_{\mathrm{ef}}\left(\frac{P_{1}}{P_{10}}\right)^{((n-1) / n)} \dot{m}_{1}+\frac{n P_{1}}{V_{1}} \dot{V}_{1} A_{\mathrm{ef}} \\
& -\left(P_{1}-P_{a}\right) \frac{\mathrm{d} A_{\mathrm{ef}}}{\mathrm{d} x} .
\end{aligned}
$$

According to formula (11), the vertical stiffness $K_{\text {dyn }}$ based on the thermodynamics can well reflect the stiffness characteristics of air spring. However, the rubber diaphragm volume $V_{1}$ and effective area $A_{\text {ef }}$ and their derivative terms cannot be clearly expressed by a mathematical formula. Thus, in this paper, these functions will be solved by approximate analysis method, as described in the following section.

2.2. Restriction Coefficient Modeling. The air spring system used on the metro vehicles is different from the typical bellow-pipe-tank pneumatic system. The auxiliary chamber is linked to rubber diaphragm via an orifice (as shown in Figure 1). The effect of pipe length, pipe friction coefficient, and air inertia in the pipe on the damping characteristics is unnecessary to consider [20], and the damping coefficient models proposed in $[11,19]$ are not suitable for the air spring system.

The orifice connects the rubber diaphragm with the auxiliary chamber; once there is a pressure difference between them, a certain restriction force can be generated during the flow through the orifice. Therefore, the air mass flow rate in the air spring is closely related to the pressure difference and orifice diameter. Referring to literature [21], the complex compressible flow through the orifice can nonetheless be described by simple incompressible-flow local head loss:

$$
\dot{m}=\pi d_{\text {or }}^{2} \sqrt{\frac{\rho \Delta P}{8 k}}
$$

where $\dot{m}$ is root mean square (RMS) mass flow rate through the orifice, $d_{\text {or }}$ is diameter of orifice, $\Delta P$ is RMS pressure difference, $\rho$ is average density, and $k$ is local head loss coefficient.

The RMS mass flow rate $\dot{m}$ can be estimated from the rubber diaphragm side (see (5)) and also can be calculated by the reservoir side (see (10)), as well as the following function:

$$
\dot{m}=a d_{\text {or }}^{b} \Delta P^{c},
$$

where parameters $a, b$, and $c$ are determined by minimizing the error between (12) and (13).

The RMS pressure difference $\Delta P$ can be obtained from (4) and (9). The local head loss coefficient $k$ varies with the orifice diameter $d_{\text {or }}$. The corresponding relationship between diameter $d_{\text {or }}$ and head loss coefficient $k$ is obtained through experimental simulation, as shown in Table 1 [21].

2.3. Geometrical Analysis of Air Spring. The geometric parameters such as the rubber diaphragm volume $V_{1}$ and effective area $A_{\text {ef }}$ and their derivative terms $\dot{V}_{1}$ and $\dot{A}_{\text {ef }}$ play a 
TABLE 1: Corresponding relationship between orifice diameter and head loss coefficient.

\begin{tabular}{lcccc}
\hline Symbol & \multicolumn{5}{c}{ Value } \\
\hline$d_{\text {or }}(\mathrm{mm})$ & 5 & 10 & 14 & 20 \\
$k$ & 0.753 & 0.637 & 0.634 & 0.954 \\
\hline
\end{tabular}

key role in the air spring vertical stiffness. In previous studies $[17,19,22]$, these geometric parameters have been usually determined by experiments. In this paper, the geometric parameters are conducted by an approximate analysis method; this is very beneficial for quantitative analysis of the relationship between stiffness characteristics and geometric parameters.

In order to obtain an analytical solution of the air spring by using the approximate analysis approach, the following simplifications and assumptions are required [5-7]:

(i) The diaphragm is assumed to be a circular arc.

(ii) The change of arc length is ignored in the process of vertical deformation.

Under a small vertical deformation $\mathrm{d} x$, the vertical deflection of the top plate and rubber diaphragm changes from the initial position to the current position (the black solid line changes to the red dotted line), as shown in Figure 2.

The symbols in Figure 2 are defined as follows: $O$ and $O_{1}$ are centers of the circular arc before and after the deflection, $R$ and $\mathrm{d} R$ are the effective radius of the air spring and its variation, and $\mathrm{d} R$ is defined to positive (or negative), when $R$ is increased (or decreased), $\phi$ is the constraint angle of the top plate, $\theta$ and $\theta+\mathrm{d} \theta$ are the angles of the diaphragm before and after deflection, and $S, \mathrm{~d} s, \mathrm{~d} x^{\prime}$, and $\mathrm{d} x^{\prime \prime}$ are intermediate variables, which can be easily understood by referring to Figure 2.

Based on the schematic diagram Figure 2 and assumption ii, the geometric variable equation of the arc length before and after deflection can be obtained as

$$
\begin{gathered}
S+\mathrm{d} x^{\prime \prime}+2 r \theta=S+\mathrm{d} s+2(r-\mathrm{d} r)(\theta+\mathrm{d} \theta), \\
\mathrm{d} x^{\prime \prime}-\mathrm{d} s=2 r \mathrm{~d} \theta-2 \theta \mathrm{d} r,
\end{gathered}
$$

where $d s$ can be derived by calculating the length of $B C$ and $B C^{\prime}$ in the triangle $A B C$ and $A^{\prime} B C^{\prime}$ as follows:

$$
\begin{aligned}
\mathrm{d} s= & 2(r-\mathrm{d} r) \sin [\pi-(\theta+\mathrm{d} \theta)] \sin \left\{\frac{\pi}{2}-[\pi-(\theta+\mathrm{d} \theta)]\right\} \\
& -2 r \sin (\pi-\theta) \sin \left[\frac{\pi}{2}-(\pi-\theta)\right],
\end{aligned}
$$

$$
\mathrm{d} s=\sin 2 \theta \mathrm{d} r-2 r \cos 2 \theta \mathrm{d} \theta .
$$

Combining (15) and (17), we obtain

$$
2 r(1-\cos 2 \theta) \mathrm{d} \theta+(\sin 2 \theta-2 \theta) \mathrm{d} r=\mathrm{d} x^{\prime \prime} .
$$

Comparing the length of $A C$ and $A C^{\prime}$ in the triangle $A B C$ and $A^{\prime} B C^{\prime}, \mathrm{d} x^{\prime}$ can be derived as follows:

$$
\mathrm{d} x^{\prime}=2 \sin ^{2} \theta \mathrm{d} r-2 r \sin 2 \theta \mathrm{d} \theta .
$$

From the deformation in Figure 2, the following relations can be found:

$$
\begin{aligned}
\mathrm{d} x^{\prime} & =\sin \phi \mathrm{d} x, \\
\mathrm{~d} x^{\prime \prime} & =\cos \phi \mathrm{d} x .
\end{aligned}
$$

Substituting (19), (20), and (21) into (18), the following equations can be derived:

$$
\begin{aligned}
\mathrm{d} r & =\frac{(1-\cos 2 \theta) \sin \phi+\sin 2 \theta \cos \phi}{4\left(\sin ^{4} \theta+\sin ^{2} \theta \cos ^{2} \theta-\theta \sin \theta \cos \theta\right)} \mathrm{d} x, \\
\mathrm{~d} \theta & =\frac{2 \sin ^{2} \theta \cos \phi-(\sin 2 \theta-2 \theta) \sin \phi}{8 r\left(\sin ^{4} \theta+\sin ^{2} \theta \cos ^{2} \theta-\theta \sin \theta \cos \theta\right)} \mathrm{d} x,
\end{aligned}
$$

where $\mathrm{d} R$ can be calculated in the triangle $O B D$ and $O^{\prime} B D^{\prime}$ as follows:

$$
\mathrm{d} R=2 r \sin (2 \theta-\phi) \mathrm{d} \theta+\cos (2 \theta-\phi) \mathrm{d} r .
$$

Substituting (22) and (23) into (24), $\mathrm{d} R$ can be expressed as follows:

$$
\mathrm{d} R=\frac{\sin (2 \theta-\phi)\left[2 \sin ^{2} \theta \cos \phi-(\sin 2 \theta-2 \theta) \sin \phi\right]+\cos (2 \theta-\phi)[(1-\cos 2 \theta) \sin \phi+\sin 2 \theta \cos \phi]}{4\left(\sin ^{4} \theta+\sin ^{2} \theta \cos ^{2} \theta-\theta \sin \theta \cos \theta\right)} \mathrm{d} x .
$$

The effective area $A_{\text {ef }}$ is defined as

$$
A_{\text {ef }}=\pi R^{2} \text {. }
$$

The effective area after deflection can be expressed as follows:

$$
\mathrm{d} A_{\mathrm{ef}}=\pi(R+\mathrm{d} R)^{2}-\pi R^{2} \approx 2 \frac{A_{\mathrm{ef}}}{R} \mathrm{~d} R .
$$

Combining (25) and (27), the rate of change of the effective area can be derived as

$$
\frac{\mathrm{d} A_{\mathrm{ef}}}{\mathrm{d} x}=\alpha \frac{A_{\mathrm{ef}}}{R},
$$

where $\alpha=\left(\sin (2 \theta-\phi) \quad\left[2 \sin ^{2} \theta \cos \quad \phi-(\sin 2 \theta-2 \theta) \sin \right.\right.$ $\phi]+\cos \quad(2 \theta-\phi)[(1-\cos 2 \theta) \sin \phi+\sin 2 \theta \quad \cos \phi] / 2$ $\left(\sin ^{4} \theta+\sin ^{2} \theta \cos ^{2} \theta-\theta \sin \theta \cos \theta\right)$. 

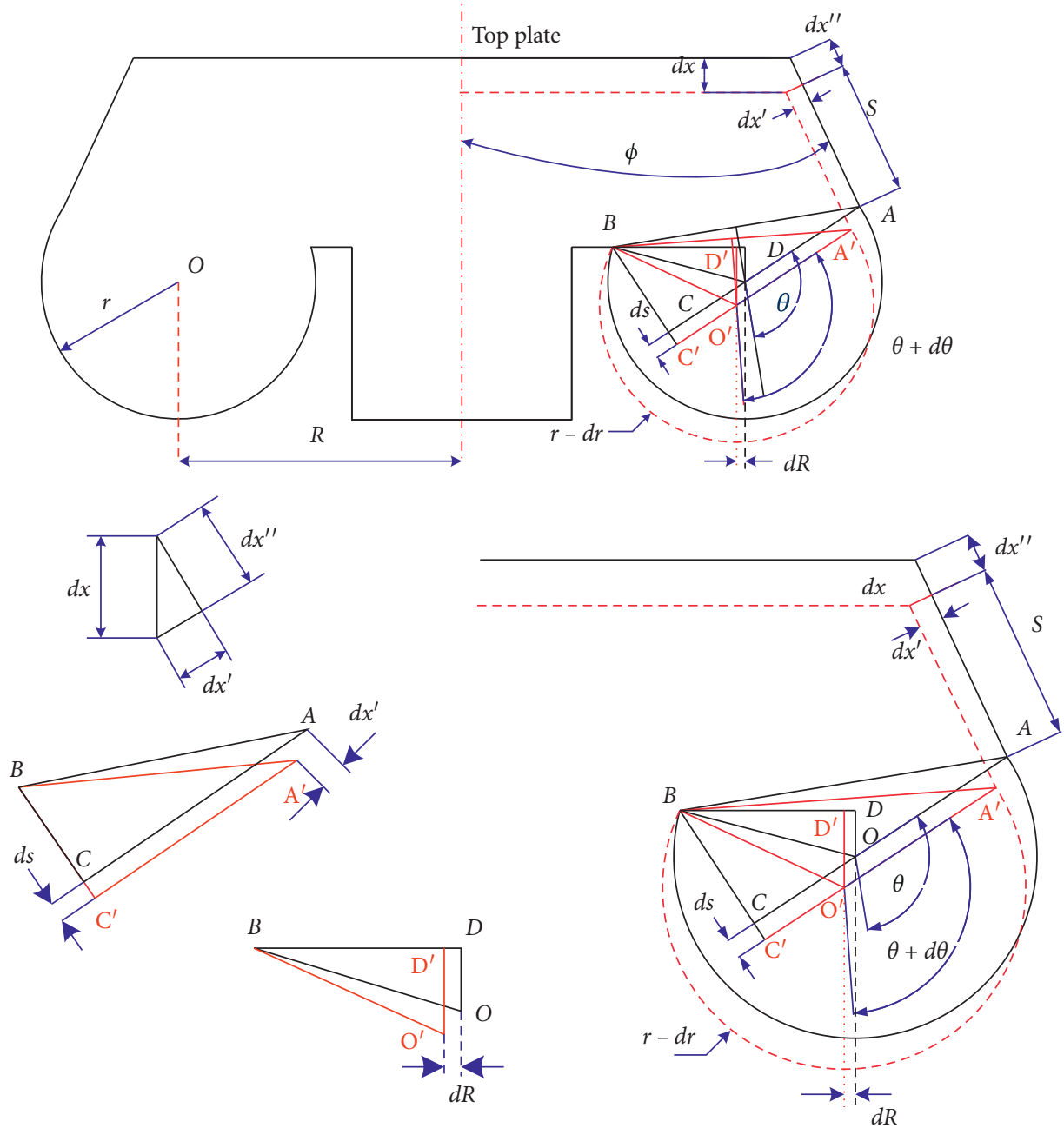

FIgURE 2: Schematic diagram of the vertical deformation of air spring.

After a small vertical deformation, the change rate of the volume in the rubber diaphragm can be expressed simplicity as follows:

$$
\frac{\mathrm{d} V_{1}}{\mathrm{~d} x} \approx A_{\mathrm{ef}}
$$

Substituting (28) and (29) into (11) yields the formula for the dynamic vertical stiffness of the air spring:

$$
\begin{aligned}
K_{\mathrm{dyn}}= & \frac{n P_{1}}{V_{1}} A_{\mathrm{ef}}^{2}-\frac{n G T_{10}}{V_{1}} A_{\mathrm{ef}}\left(\frac{P_{1}}{P_{10}}\right)^{((n-1) / n)}\left(\frac{\mathrm{d} \rho_{1}}{\mathrm{~d} t} V_{1}+\frac{\mathrm{d} V_{1}}{\mathrm{~d} t} \rho_{1}\right) \\
& -\alpha\left(P_{1}-P_{a}\right) \frac{A_{\mathrm{ef}}}{R},
\end{aligned}
$$

where $\alpha=\left(\sin (2 \theta-\phi)\left[2 \sin ^{2} \theta \cos \phi-(\sin 2 \theta-2 \theta) \sin \phi\right]+\right.$ $\cos (2 \theta-\phi)[(1-\cos 2 \theta) \sin \phi+\sin 2 \theta \cos$

$\left.\phi] / 2\left(\sin ^{4} \theta+\sin ^{2} \theta \cos ^{2} \theta-\theta \sin \theta \cos \theta\right)\right)$.

\section{Experiments and Simulation}

The above analytical calculation was performed on ZMC080-J air spring, which was designed and manufactured by the CRRC ZhuZhou Locomotive Company. The profile and dimension of this air spring are shown in Figure 3, and the main geometric parameters are listed in Table 2.

A series of experiments were also carried out at the CRRC ZhuZhou Locomotive Company. The schematic diagram of the test bench for the vertical stiffness performance test of air spring is shown in Figure 4.

The structure and working principle of the test bench as shown in Figure 4 have been described in detail in $[1,6,23]$. A linear actuator was positioned at the bottom of the test bench to represent a sinusoidal excitation, a load sensor was mounted in the upper part of the setup to measure the vertical force, and pressure sensors were applied to monitor the internal pressure of the air spring. A pressure sensor is installed at the junction of the rubber diaphragm and the pipe to detect the pressure of the rubber diaphragm, and the pressure of the auxiliary chamber is monitored at the junction of the auxiliary chamber and the pipe, as shown in Figure 4(b). The sensor parameters are as follows:

Pressure sensor with working voltage 5-12 VDC, measuring range $0-300 \mathrm{MPa}$, working temperature $-20^{\circ}-120^{\circ}$, and measuring accuracy $0.25 \%$ FS; laser displacement sensor with working voltage $12-24 \mathrm{VDC}$, measuring range 


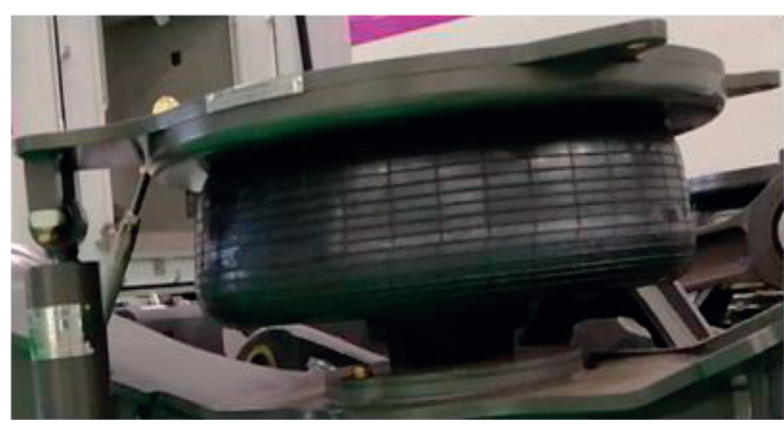

(a)

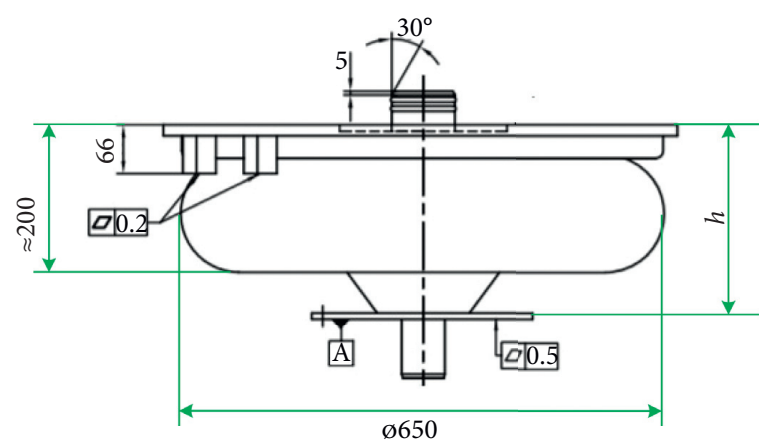

(b)

Figure 3: Profile and dimension of ZMC080-J air spring.

TABLE 2: Geometric parameters of ZMC080-J air spring.

\begin{tabular}{lcc}
\hline Symbol & Description & Value \\
\hline$d_{\text {or }}$ & Diameter of orifice & $14 \mathrm{~mm}$ \\
$h$ & Standard height of air spring & $262 \mathrm{~mm}$ \\
$\phi$ & Constraint angle of top plate & $55^{\circ}$ \\
$\theta$ & Angle of diaphragm & $120^{\circ}$ \\
\hline
\end{tabular}

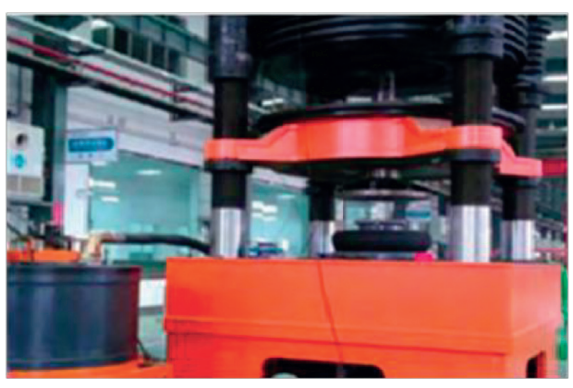

(a)

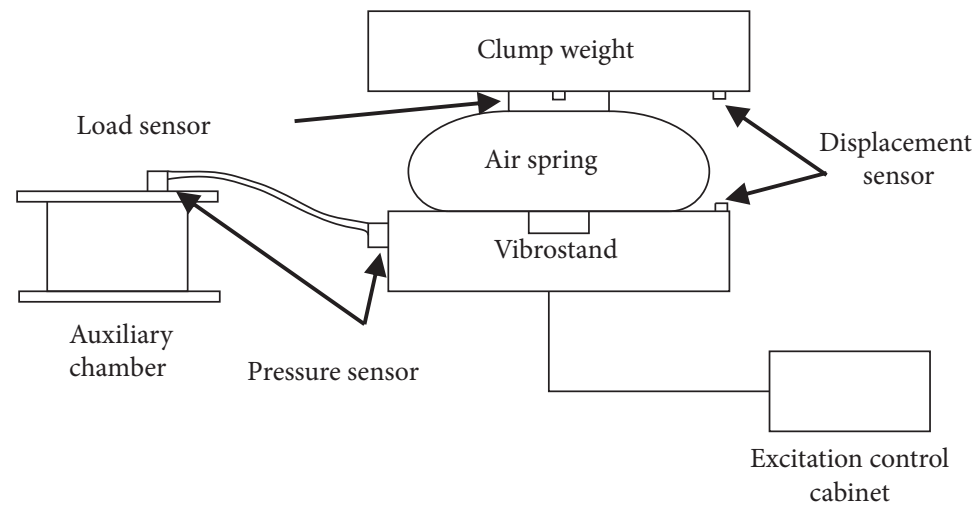

(b)

FIgURE 4: Schematic diagram of air spring test bench.

0-100 mm, resolution $0.07 \mathrm{~mm}$, and linearity $\pm 0.1 \% \mathrm{FS}$; load sensor with working voltage $5-12 \mathrm{VDC}$, measuring range $20 \mathrm{Kg}-30 \mathrm{~T}$, accuracy $\leq 0.05 \%$, and sensitivity $2.0 \pm 10 \%$ $\mathrm{mV} / \mathrm{V}$.

3.1. Experimental Data. The dynamic experiments of air spring were carried out on the basis of the EN13597-2003 Railway applications-Rubber suspension components-Rubber diaphragms for pneumatic suspension springs [24] and TBT2841/2010 Air spring for railway vehicles [25]. The vertical preload on air spring was $53.7 \mathrm{KN}$ to 96.9 KN, representing no-load case AW0 and overload case AW3, respectively. The vertical displacement excitation was simulated by a sinusoidal wave with a frequency range of $0 \mathrm{~Hz}$ to $20 \mathrm{~Hz}$ and an amplitude range of $2 \mathrm{~mm}$ to $10 \mathrm{~mm}$.

The main parameters for the air spring test bench are summarized in Table 3.
TABLE 3: Main parameters for air spring test bench.

\begin{tabular}{lc}
\hline Parameter & Value \\
\hline Vertical preload & $53.7 \mathrm{KN}-177 \mathrm{KN}$ \\
Diaphragm volume $V_{1}$ & $\approx 32.5 \mathrm{~L}$ \\
Auxiliary chamber volume $V_{2}$ & $0 \mathrm{~L}-150 \mathrm{~L}$ \\
Length of pipe $L_{p}$ & $1.25 \mathrm{~m}$ \\
Diameter for pipe $d_{p}$ & $38 \mathrm{~mm}$ \\
Excitation amplitude & $2 \mathrm{~mm}-10 \mathrm{~mm}$ \\
Excitation frequency & $0 \mathrm{~Hz}-20 \mathrm{~Hz}$ \\
\hline
\end{tabular}

When air spring is subjected to an excitation wave with a frequency of $0.125 \mathrm{~Hz}$ and an amplitude of $10 \mathrm{~mm}$, the stiffness-vertical load curves at various auxiliary chamber volumes for air spring are shown in Figure 5(a). The vertical stiffness increases linearly with the increase of the vertical load and decreases with the increase of the auxiliary chamber volume. 


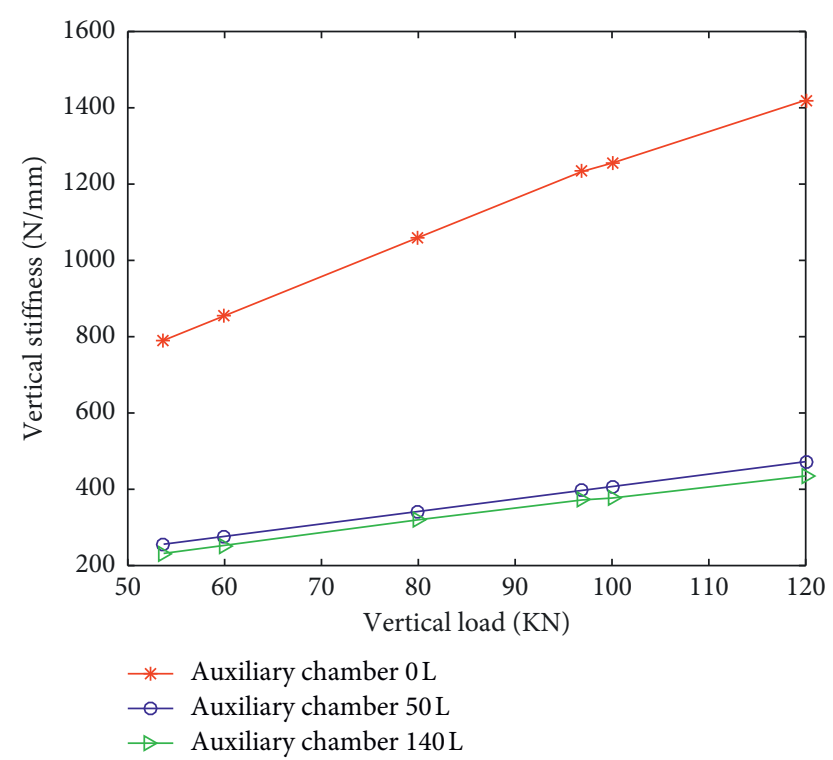

(a)

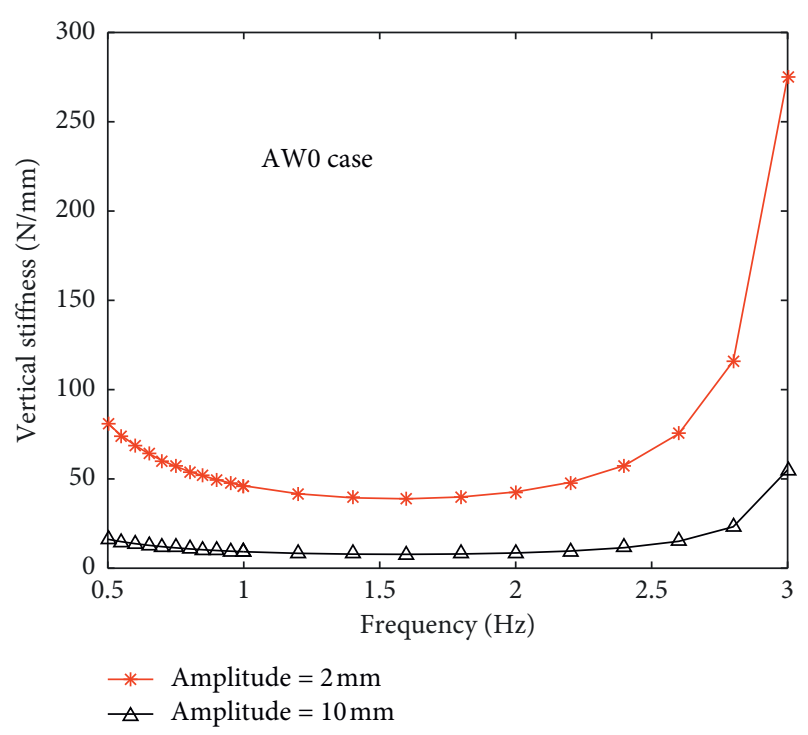

(b)

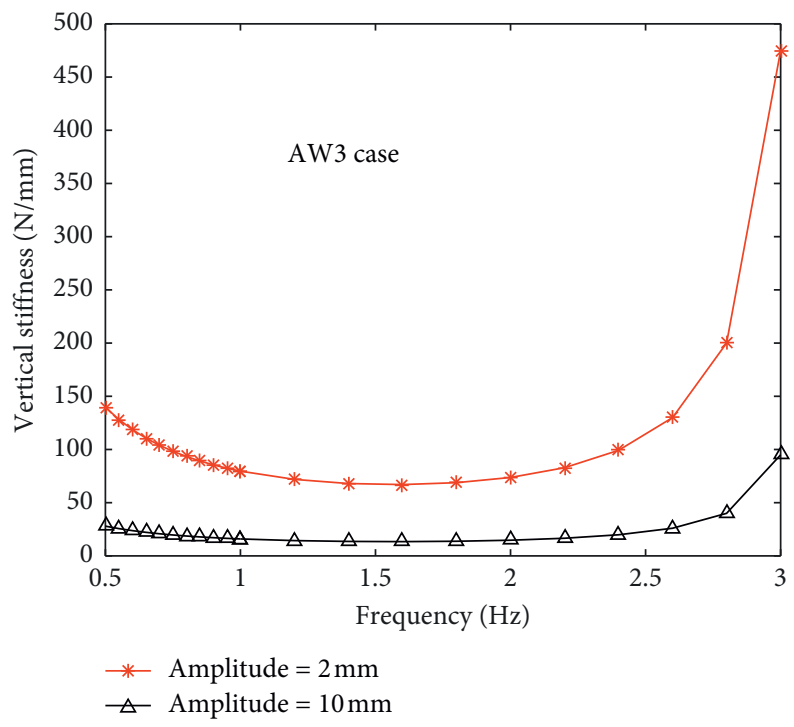

(c)

Figure 5: Experimental data.

Furthermore, when the auxiliary chamber volume is $50 \mathrm{~L}$, the relationship between the vertical stiffness and the excitation amplitude and frequency under the case of AWO and AW3 is shown in Figure 5(b) and 5(c). The vertical stiffness increases with the increase of frequency and decreases with the increase of amplitude.

3.2. Simulation and Validation. Under the same conditions as the experiment, the excitation wave with a frequency of $0.125 \mathrm{~Hz}$, and an amplitude of $10 \mathrm{~mm}$, the comparison results of the dynamic vertical stiffness model with and without auxiliary chamber are shown in Figure 6. It is clear that both the experimental data and simulation results reflect the tendency that the vertical stiffness increases almost linearly with the increase of the vertical load, but there is a deviation about $5 \%$ between them; the reason for this behavior is that the analytical solution of the geometric parameters is derived based on the above simplifications and assumptions. It also can be seen that when the auxiliary chamber volume $V_{2}$ is $50 \mathrm{~L}$, the vertical stiffness of the air spring which has a significant reduction of about $70 \%$ compared with the simulation result of the auxiliary chamber is $0 \mathrm{~L}$. Therefore, the comparison results indicate that the proposed vertical stiffness model can well characterize the dynamic characteristics of the air spring system 


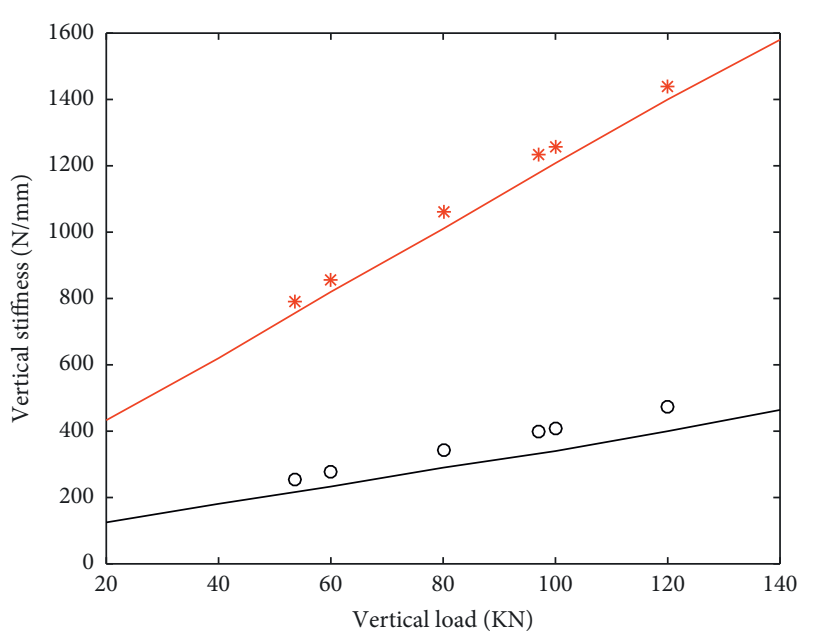

* Experimental data auxiliary chamber $0 \mathrm{~L}$
— Experimental data auxiliary chamber $50 \mathrm{~L}$
— Simulation results auxiliary chamber $0 \mathrm{~L}$
_ Simulation results auxiliary chamber $50 \mathrm{~L}$

Figure 6: Comparison results of the proposed model and experimental data.

and also confirm that the vertical stiffness of the air spring decreases with the increase of the auxiliary chamber volume.

\section{Analysis and Discussions}

From a qualitative point of view, sensitivity analysis is a useful tool for investigating the individual influence of design parameters on the properties of the product and offering guidelines for product manufacture. In this section, under the situation of AW3 and subjected to an excitation amplitude of $10 \mathrm{~mm}$, the impact of the geometric parameters on the vertical stiffness characteristics of the air spring is analyzed using a sensitivity analysis approach.

4.1. Impact of Rubber Diaphragm Volume. From (30), the vertical stiffness of the air spring is mainly contributed by the internal pressure and the volume of rubber diaphragm. The influence of the diaphragm volume on the vertical stiffness is shown in Figure 7.

When the auxiliary chamber is $0 \mathrm{~L}$, the vertical stiffness decreases dramatically as the diaphragm volume increases, and the steep curve becomes flattened when the diaphragm chamber volume exceeds $30 \mathrm{~L}$, as shown in Figure 7(a). However, when the rubber diaphragm is connected with an auxiliary chamber with a volume of $50 \mathrm{~L}$, the recession curve of the vertical stiffness becomes smooth with the increase of the chamber volume, as shown in Figure 7(b). This is because the damping produced by the gas through the orifice effectively alleviates the attenuation of the vertical stiffness caused by the increase of the rubber diaphragm volume. Therefore, it is verified again that the auxiliary chamber has the characteristics of regulating the vertical stiffness of air spring.
4.2. Impact of Auxiliary Chamber Volume. Figure 8 shows that the auxiliary chamber volume has a significant effect on regulating the vertical stiffness, the vertical stiffness decreases dramatically as the auxiliary chamber volume increases up to $60 \mathrm{~L}$, and the recession curve becomes less steep as the chamber volume exceeds $60 \mathrm{~L}$. Combined with Figure 7(b), a reasonable volume ratio of the rubber diaphragm to the auxiliary chamber is needed to make full use of the damping characteristics of the orifice. Therefore, in order to set an appropriate volume for the auxiliary chamber, referring to literature [5], the following equation can be derived from (30):

$$
V_{2}>\frac{n P_{1} R_{0} A_{\mathrm{ef}}}{\alpha\left(P_{1}-P_{a}\right)}-V_{1} .
$$

4.3. Impact of Orifice Diameter. Figures 9(a) and 9(b) show the influence of orifice diameter on the vertical stiffness and mass flow rate at different orifice diameter $5 \mathrm{~mm}, 14 \mathrm{~mm}$, and $20 \mathrm{~mm}$.

As can be observed from Figure 9(a) that the vertical stiffness of air spring increases monotonously with the increase of frequency and decreases with increasing orifice diameter. This can be mainly attributed to the fact that low frequencies and large orifice diameters allow enough time to let air flow through the orifice. On the contrary, higher frequencies and smaller orifice diameters would limit the air flow through the orifice so as to increase the inner pressure of rubber diaphragm, which leads to the vertical stiffness increases.

It can be seen from Figure 9(b) that, with the increase of orifice diameter and pressure difference, the mass flow rate increases, but the smaller the orifice, the smoother the mass flow rate changes with the increase of pressure difference. The reason is the same as that in Figure 9(a); large orifice diameter and large pressure difference allow more air flow through the orifice; conversely, a smaller orifice diameter limits the mass flow through the orifice.

According to the influence of orifice diameter on the vertical stiffness and mass flow rate, the optimal orifice size can be designed quickly.

4.4. Impact of Shape Coefficient. Figure 10(a) shows the relationship between vertical stiffness and the shape coefficient $\alpha$; it is clear that the vertical stiffness decreases with the shape coefficient increases. The shape coefficient $\alpha$ consists of the constraint angle of the top plate $\phi$ and the angle of the diaphragm $\phi$. The relationship between the constraint angle $\phi$, the angle of the diaphragm $\theta$, and the shape coefficient $\alpha$ is shown in Figure 10(b). Under the synergistic effect of the constraint angle $\phi$ and angle $\theta$, with the increase of constraint angle $\phi$ and the decrease of angle $\theta$, the shape coefficient $\alpha$ increases. Figure 10(c) shows that the shape coefficient $\alpha$ decreases as the angle $\theta$ increases from $45^{\circ}$ to $165^{\circ}$. The relationship between the shape coefficient $\alpha$ and the constraint angle $\phi$ is shown in Figure 10(d); the shape coefficient $\alpha$ first decreases and then increases as the 


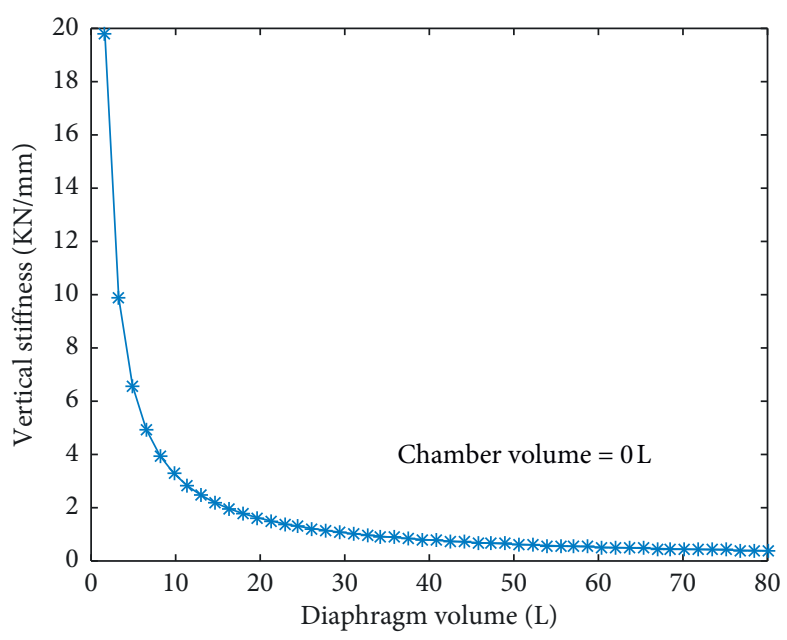

(a)

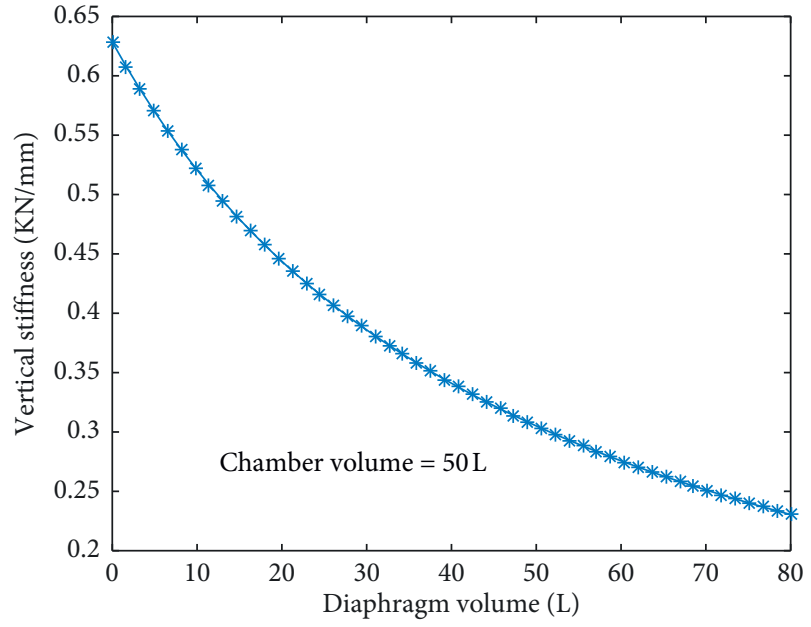

(b)

Figure 7: Impact of rubber diaphragm volume on vertical stiffness.

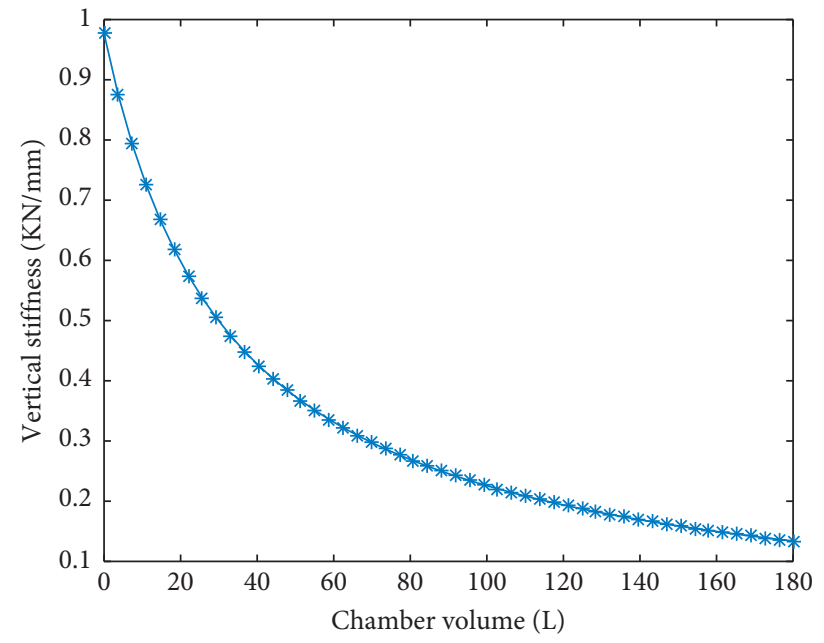

FIGURE 8: Impact of auxiliary chamber volume on vertical stiffness.

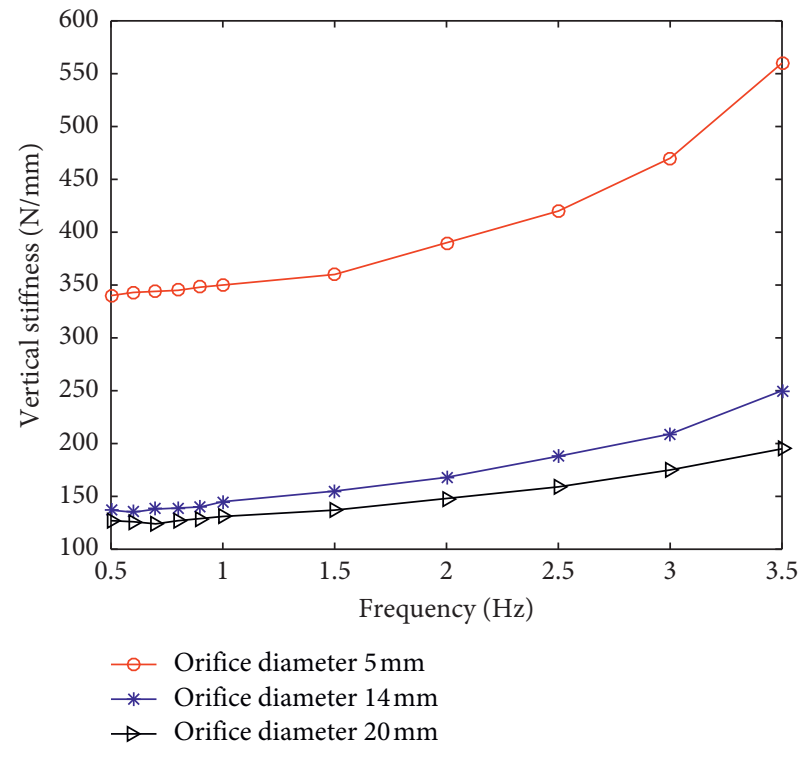

(a)

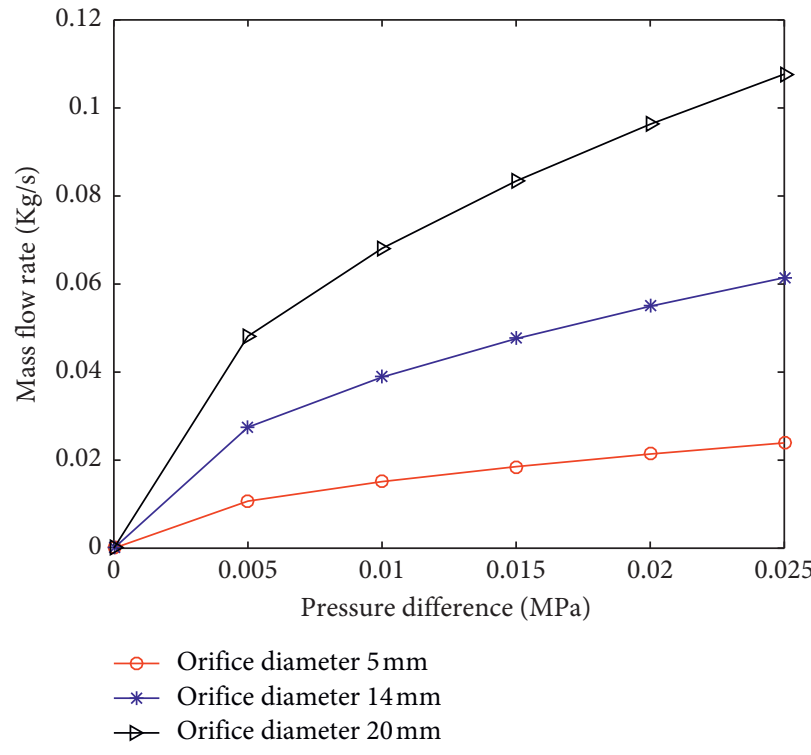

(b)

FIgURE 9: Impact of orifice diameter on stiffness and mass flow rate. 


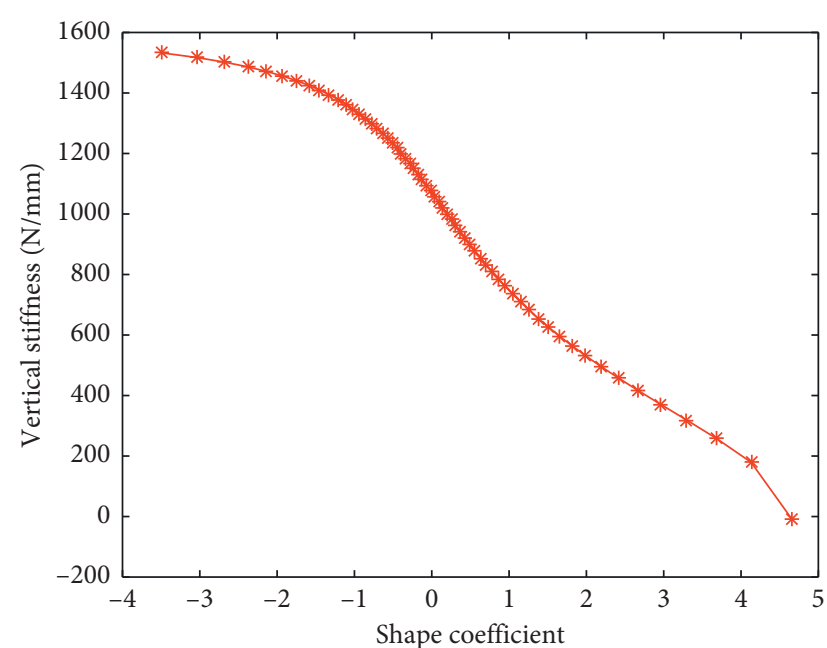

(a)

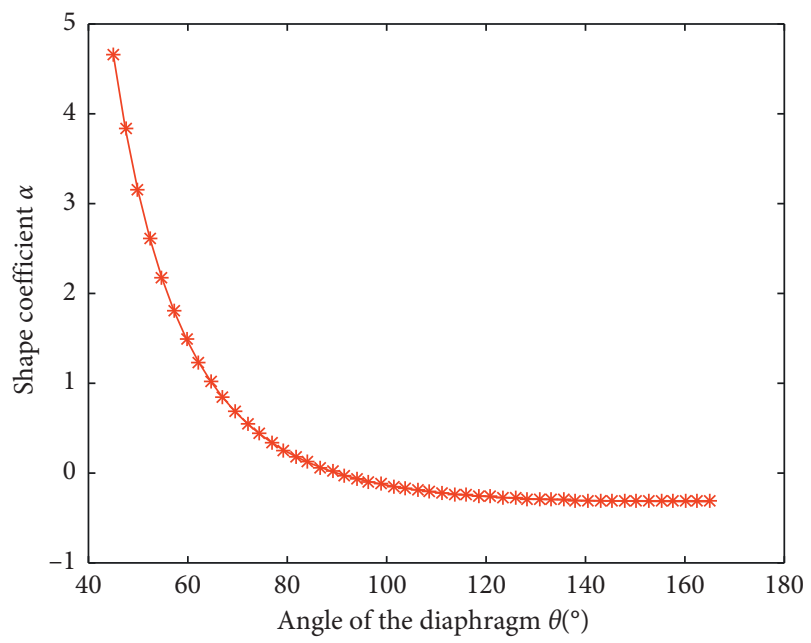

(c)

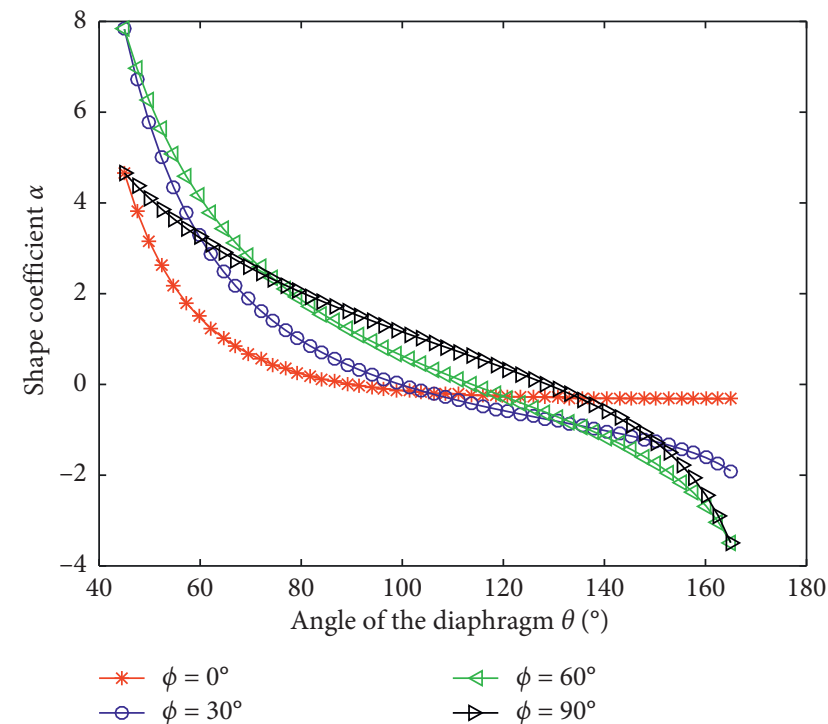

(b)

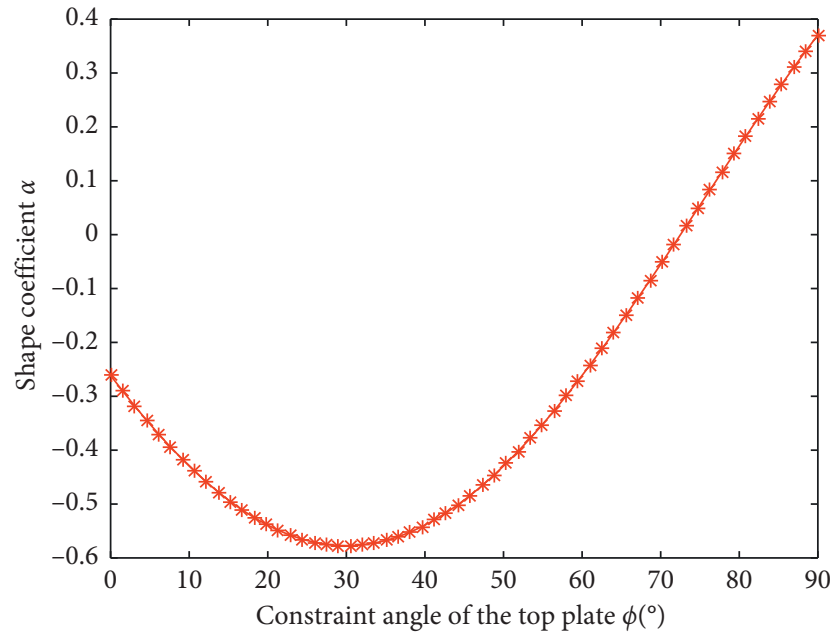

(d)

FIGURE 10: Impact of shape coefficient on vertical stiffness.

constraint angle $\phi$ increases from $0^{\circ}$ to $90^{\circ}$, and a minimum value appears at the inflection point of $30^{\circ}$.

According to the relationship between the vertical stiffness and the shape coefficient, if the vertical stiffness needs to be increased, the shape coefficient can be designed to be smaller than zero; this means that a reasonable range of the angle of diaphragm $\theta$ and the constraint angle $\phi$ is $\left(100^{\circ} \sim 165^{\circ}\right)$ and $\left(70^{\circ} \sim 90^{\circ}\right)$. Conversely, if the stiffness needs to be decreased, the shape coefficient can be designed to be greater than zero, which means that the range of the angle of diaphragm $\theta$ and the constraint angle $\phi$ is $\left(45^{\circ} \sim 100^{\circ}\right)$ and $\left(0^{\circ} \sim 70^{\circ}\right)$.

In general, in terms of the above discussion, it helps designers to quickly estimate the shape coefficient of air springs.

\section{Conclusions and Future Work}

In this study, a dynamic vertical stiffness model for free membrane-type air spring was constructed based on thermodynamics and fluid dynamics, and the geometric parameters were processed with an approximate analytical method. Meanwhile, air spring performance experiments were carried out to verify the accuracy and reliability of the proposed model. Furthermore, the impact of geometric parameters of the air spring such as the rubber diaphragm volume, auxiliary chamber volume, orifice diameter, and shape coefficient on the vertical stiffness characteristics was analyzed using the sensitivity analysis method, and some valuable conclusions are drawn as follows: 
(i) The dynamic vertical stiffness model of the diaphragm-orifice-chamber system is successfully established on the basis of the thermodynamic, fluid-dynamic theory to reproduce its mechanical characteristics.

(ii) The analytical solutions of the effective area, the equivalent volume, and their derivative terms are obtained by the approximate analytical method.

(iii) A reasonable volume ratio of the rubber diaphragm to the auxiliary chamber can be obtained in terms of formula (31).

(iv) According to the influence of orifice diameter on the vertical stiffness and mass flow rate, the optimal orifice size can be designed.

(v) Based on the relationship between the vertical stiffness and the shape coefficient, it can help designers to quickly estimate the shape coefficient of air springs.

In summary, the dynamic vertical stiffness model can accurately predict the mechanical characteristics of the air spring system and also provide a guidance for designing the parameters as well as for matching the air spring dynamic performance.

For future studies, the damping force generated by air flow through the orifice will be studied, and the relationship between the damping characteristics and various factors will be the focus of the ongoing research.

\section{Data Availability}

The experimental data used to support the findings of this study are available from the corresponding author upon request.

\section{Conflicts of Interest}

The authors declare that there are no conflicts of interest regarding the publication of this paper.

\section{Acknowledgments}

The author received no financial support for the research, authorship, and/or publication of this article.

\section{References}

[1] F. Li and Z. Qi, "Application and research of air spring suspension system for rail vehicle," China Railway, vol. 4, pp. 42-47, 2014.

[2] A. Alonso, J. G. Giménez, J. Nieto, and J. Vinolas, "Air suspension characterisation and effectiveness of a variable area orifice," Vehicle System Dynamics, vol. 48, no. 1, pp. 271-286, 2010.

[3] A. Genovese, S. Strano, and M. Terzo, "Model-based study of a resonant system in a railway air spring secondary suspension for energy harvesting applications," in Proceedings of the 2018 2nd International Conference on Mechatronics Systems and Control Engineering, ACM, Amsterdam, Netherlands, February 2018.
[4] W. K Shi, W. Jiang, Y. Huang et al., "Finite element analysis of an air spring concerning initial pressure and parameters of cord fabric layer," in Proceedings of the second Asia-Pacific Conference on Computational Intelligence and Industrial Applications, IEEE, Los Alamitos, CA, USA, pp. 496-499, November 2009.

[5] X. Li and T. Li, "Research on vertical stiffness of belted air springs," Vehicle System Dynamics, vol. 51, no. 11, pp. 1655-1673, 2013.

[6] X. B. Li, Y. He, W. Q. Liu et al., "Research on vertical stiffness of a rolling lobe air spring," Journal of Rail and Rapid Transit, vol. 230, no. 4, pp. 1172-1183, 2015.

[7] J. J. Chen, Z. H. Yin, S. Rakheja et al., “Theoretical modeling and experimental analysis of the vertical stiffness of a convoluted air spring including the effect of the stiffness of the bellows," Proceedings of the Institution of Mechanical Engineers, Part D: Journal of Automobile Engineering, vol. 232, no. 4, pp. 547-561, 2017.

[8] H. Liu and J. C. Lee, "Model development and experimental research on an air spring with auxiliary reservoir," International Journal of Automotive Technology, vol. 12, no. 6, pp. 839-847, 2011.

[9] G. Quaglia and M. Sorli, "Air suspension dimensionless analysis and design procedure," Vehicle System Dynamics, vol. 35, no. 6, pp. 443-475, 2001.

[10] G. Quaglia and A. Guala, "Evaluation and validation of an air spring analytical model," International Journal of Fluid Power, vol. 4, no. 2, pp. 43-54, 2003.

[11] N. Docquier, P. Fisette, and H. Jeanmart, "Multiphysic modelling of railway vehicles equipped with pneumatic suspensions," Vehicle System Dynamics, vol. 45, no. 6, pp. 505-524, 2007.

[12] T. Nakajima, Y. Shimokawa, M. Mizuno et al., "Air suspension system model coupled with leveling and differential pressure valves for railroad vehicle dynamics simulation," Journal of Computational and Nonlinear Dynamics, vol. 9, pp. 1-9, 2014.

[13] H. Zhu, J. Yang, Y. Zhang, and X. Feng, "A novel air spring dynamic model with pneumatic thermodynamics, effective friction and viscoelastic damping," Journal of Sound and Vibration, vol. 408, pp. 87-104, 2017.

[14] A. Facchinetti, L. Mazzola, S. Alfi, and S. Bruni, "Mathematical modelling of the secondary airspring suspension in railway vehicles and its effect on safety and ride comfort," Vehicle System Dynamics, vol. 48, no. 1, pp. 429-449, 2010.

[15] J. S. Wang and S. H. Zhu, "Research on linear model about dynamic stiffness of air spring with auxiliary chamber," Journal of Vibration and Shock, vol. 2, pp. 72-78, 2009.

[16] S. Bruni, J. Vinolas, M. Berg, O. Polach, and S. Stichel, "Modelling of suspension components in a rail vehicle dynamics context," Vehicle System Dynamics, vol. 49, no. 7, pp. 1021-1072, 2011.

[17] P. Pintado, C. Ramiro, M. Berg et al., "On the mechanical behavior of rubber springs for high speed rail vehicles," Journal of Vibration and Control, vol. 24, no. 20, pp. 46764688, 2018.

[18] Z. Qi, F. Li, and D. L. Yu, "A three-dimensional coupled dynamics model of the air spring of a high-speed electric multiple unit train," Proceedings of the Institution of $\mathrm{Me}$ chanical Engineers, Part: Journal of Rail and Rapid Transit, vol. 231, no. 1, pp. 3-18, 2015.

[19] A. J. Nieto, A. L. Morales, A. González, J. M. Chicharro, and P. Pintado, "An analytical model of pneumatic suspensions 
based on an experimental characterization," Journal of Sound and Vibration, vol. 313, no. 1, pp. 290-307, 2008.

[20] T. Katsuya, Y. Chuuji, K. Toshiharu et al., "Study on dynamic characteristic análisis of air spring with auxiliary chamber," JSAE Review, vol. 20, pp. 349-355, 1999.

[21] P. Pintado, C. Ramiro, A. J. Nieto et al., "The dynamic behavior of pneumatic vibration isolators," Journal of Vibration and Control, vol. 24, no. 20, pp. 4563-4574, 2018.

[22] N. Doquier, Multiphysics modeling of multibody system$s$-application to railway pneumatic suspensions, Ph.D. thesis, Université Catholique de Louvain, Ottignies-Louvain-laNeuve, Belgium, 2010.

[23] Y. Feng, M. R. Chi, W. F Qi et al., "Effect of the additional air chambers on the dynamical characteristics of air spring," Rolling Stock, vol. 56, no. 2, pp. 1-5, 2018.

[24] EN13597-2003, Railway Applications. Rubber Suspension Components. Rubber Diaphragms for Pneumatic Suspension Springs, BSI Standards, Brussels, Belgium, 2003.

[25] TB/T2841/2010, Air Spring for Railway Vehicle, China Railway Press, Beijing, China, 2010. 